\title{
The canonical projection between the shift space of an IIFS and its attractor as a fixed point
}

\section{Alexandru Mihail*}

\section{"Correspondence:}

mihail_alex@yahoo.com

Faculty of Mathematics and

Computer Science, University of

Bucharest, Str. Academiei 14,

Bucharest, 010014, Romania

\section{Springer}

\begin{abstract}
An important class of fractal sets is given by the attractors of iterated function systems which are defined as the fixed points of the associated fractal operators. In the study of such an attractor, an important place is taken by the canonical projection between the shift space associated with the system and the attractor. In this paper, by using different fixed point theorems, we present the canonical projection as the fixed point of a certain operator defined on the space of continuous functions from the shift space on the metric space associated with the system.
\end{abstract}

MSC: $54 \mathrm{H} 25 ; 28 \mathrm{~A} 80 ; 37 \mathrm{C} 70$

Keywords: IFS; IIFS; shift space; attractor; canonical projection; fixed point

\section{Introduction}

It is well known that some important mathematical objects, such as Cantor set, Sierpinsky gasket and carpet, Menger cube and the graph of Weierstrass function, are fractal sets. Most of them could be obtained as attractors of iterated function systems or of infinite iterated function systems (as in the case of Lipscomb's space, see [1-3]), that is, they can be seen as fixed points of the fractal operator associated to the corresponding (infinite) iterated function system. For iterated function system we will use the abbreviation IFS and for infinite iterated function system the abbreviation IIFS. The study of IFSs was initiated by Moran [4], clarified by Hutchinson [5] and popularized by Barnsley [6], and it has been extended by a lot of other mathematicians. There are two main directions to extend the notion of IFS and to study the attractors of such systems. The first one deals with general types of IFSs such as IIFSs, graph-directed IFSs, multifunctions iterated systems (see [7]) and generalized IFSs (see [8-10]). The second one uses different types of fixed point theorems to define the attractor of an IFS or IIFS (see [11]). As a recent example, see [12].

The fixed point theorems are used not only in the definition of the attractor of an IFS, but also for the study of its properties. For example, let us mention the case of the connectivity of the attractor (see $[13,14])$ and the very important concept associated with an IFS with probabilities, namely that of Hutchinson measure. More precisely, Hutchinson [5] proved that given $\varphi_{1}, \varphi_{2}, \ldots, \varphi_{m}$ contractions in a complete metric space $X$, there exists a unique Borel measure $\mu$ satisfying the equation $\mu=\sum_{i=1}^{m} p_{i} \mu \circ \varphi_{i}^{-1}:=M_{\mathcal{S}} \mu$, where $p_{1}, p_{2}, \ldots, p_{m}$

(c) 2015 Mihail. This article is distributed under the terms of the Creative Commons Attribution 4.0 International License (http://creativecommons.org/licenses/by/4.0/), which permits unrestricted use, distribution, and reproduction in any medium, provided you give appropriate credit to the original author(s) and the source, provide a link to the Creative Commons license, and indicate if changes were made. 
are positive numbers such that $\sum_{i=1}^{m} p_{i}=1$ and $\mathcal{S}$ is the IFS $\left(X,\left(\varphi_{i}\right)_{i \in\{1,2, \ldots, n\}}\right)$. Moreover, $\mu$ is the fixed point of the operator $M_{\mathcal{S}}$ in the topology of weak convergence within the space $\mathcal{M}$ of Borel regular probability measures with bounded support.

The shift (or code) space of an IFS and the addresses of the points lying on the attractor of the IFS are very good tools to get a more precise description of the invariant dynamics of the IFS and to study the topological properties of the attractor of the IFS. The theory of fractals tops (see [15]) provides a useful mapping from an IFS attractor into the associated code space, and it may be applied to assign colors to the IFS attractor via the method introduced by Barnsley and Hutchinson (which they refer to as color-stealing) and to construct homeomorphisms between attractors (roughly speaking, if the symbolic dynamic systems associated with the tops of two IFSs are topologically conjugate, then the attractors of the IFSs are homeomorphic). Moreover, Barnsley [15] proved that if two hyperbolic IFS attractors are homeomorphic, then they have the same entropy.

In [16] a generalization of the notion of the shift space associated to an IFS is presented. More precisely, the shift space for IIFSs is presented and the relation between this space and the attractor of the IIFS is described. A canonical projection (which turns out to be continuous) from the shift space of an IIFS on its attractor is constructed.

In this paper we provide an alternative characterization of the above mentioned projection. More precisely, by using different fixed point theorems, we present the canonical projection between the shift space and the attractor of an IIFS as a fixed point of an operator on the space of continuous functions from the shift space on the metric space associated with the system.

The paper is organized in five sections. The second one contains some preliminaries concerning fixed point theorems and classical results concerning IIFS, the third is dedicated to the main results, the fourth deals with the case of $\varepsilon$-chainable metric spaces and the last one contains some remarks and examples.

\section{Preliminaries}

For the very beginning we present some basic notations and definitions.

An increasing and right continuous function $\phi:[0, \infty) \rightarrow[0, \infty)$ such that $\phi(r)<r$ for every $r>0$ is called comparison function.

Given a function $f: X \rightarrow X, f^{[n]}$ denotes the function $f \circ f \circ \cdots \circ f$ for $n$ times.

For two metric spaces $\left(X_{1}, d_{1}\right)$ and $\left(X_{2}, d_{2}\right), C\left(X_{1}, X_{2}\right)$ denotes the set of continuous functions from $X_{1}$ into $X_{2}$ and $\mathrm{CB}\left(X_{1}, X_{2}\right)$ is the set of continuous and bounded functions from $X_{1}$ into $X_{2}$. For a function $f: X_{1} \rightarrow X_{2}, \operatorname{Lip}(f)$ denotes its Lipschitz constant, that is, $\operatorname{Lip}(f):=\sup _{x, y \in X_{1} ; x \neq y} \frac{d_{2}(f(x), f(y))}{d_{1}(x, y)}$.

Given a subset $A$ of a metric space $(X, d)$, by $d(A)$ we understand the diameter of $A$, that is, $d(A):=\sup _{x, y \in A} d(x, y)$.

For a metric space $(X, d)$, by $K(X)$ we denote the set of compact nonvoid subsets of $X$, by $B(X)$ the set of bounded closed and nonvoid subsets of $X$ and, for a set $J$, by $B_{J}(X)$ the set of bounded, closed and nonvoid subsets of $X$ having a dense subset with cardinal less or equal to the cardinal of $J$.

Note that $K(X), B_{J}(X)$ and $B(X)$ can be seen as metric spaces with the HausdorffPompeiu distance $h$ defined by

$$
h(Y, Z):=\max \left\{\sup _{y \in Y},\left(\inf _{z \in Z} d(y, z)\right), \sup _{z \in Z},\left(\inf _{y \in Y} d(y, z)\right)\right\} .
$$


Recall that $(K(X), h),\left(B_{J}(X), h\right)$ and $(B(X), h)$ are complete metric spaces if $(X, d)$ is such and compact metric spaces if $(X, d)$ is such.

Definition 2.1 Let $(X, d)$ be a metric space. A family of continuous functions $\left(f_{i}\right)_{i \in I}$, where $f_{i}: X \rightarrow X$, which is bounded (i.e., if $Y \subset X$ is bounded, then $\bigcup_{i \in I} f_{i}(Y)$ is bounded) is called an IIFS and it is denoted by $\mathcal{S}$. If the set $I$ is finite, we have an IFS. The function $F_{\mathcal{S}}: B(X) \rightarrow B(X)$ defined by $F_{\mathcal{S}}(Y)=\overline{\bigcup_{i \in I} f_{i}(Y)}$ for every $Y \in B(X)$ is called the fractal operator associated with the IIFS $\mathcal{S}$. If $Y \in K(X)$ and $I$ is finite, then $F_{\mathcal{S}}(Y)=\bigcup_{i \in I} f_{i}(Y)$.

We remark that $F_{\mathcal{S}}(K(X)) \subset K(X)$ if $I$ is a finite set and $F_{\mathcal{S}}\left(B_{I}(X)\right) \subset B_{I}(X)$ in general.

Now we recall some contractive conditions that are used in metric fixed point theorems. These are the main contractive conditions which are inherited by the fractal operator from the constitutive functions of the IFS or IIFS (as far as we know).

For a function $f: X \rightarrow X$, where $(X, d)$ is a metric space, we consider the following contractive conditions:

(i) $f$ is a contraction if there exists $a \in[0,1)$ such that $d(f(x), f(y)) \leq a d(x, y)$ for every $x, y \in X$.

(ii) $f$ is a $\phi$-contraction if $d(f(x), f(y)) \leq \phi(d(x, y))$ for every $x, y \in X$, where $\phi$ is a comparison function.

(iii) $f$ is Meir-Keeler if for every $\varepsilon>0$ there exists $\eta>0$ such that for every $x, y \in X$ with the property that $d(x, y) \leq \varepsilon+\eta$ we have $d(f(x), f(y))<\varepsilon$.

(iv) $f$ is called contractive if $d(f(x), f(y))<d(x, y)$ for every $x, y \in X, x \neq y$.

It is known (see [11] for more details) that (i) $\Rightarrow$ (ii) $\Rightarrow$ (iii) $\Rightarrow$ (iv), and we have the following theorem.

Theorem 2.2 (see [17]) Iff $: X \rightarrow X$ is Meir-Keeler, where $(X, d)$ is a complete metric space, then there exists a unique fixed point $x_{0}$ off and the sequence $\left(f^{[n]}(x)\right)_{n}$ is convergent to $x_{0}$ for every $x \in X$. If $(X, d)$ is compact and $f$ is contractive, then there exists a unique fixed point $x_{0}$ off and the sequence $\left(f^{[n]}(x)\right)_{n}$ is convergent to $x_{0}$ for every $x \in X$.

Definition 2.3 The family of functions $\left(f_{i}\right)_{i \in I}$, where $f_{i}: X \rightarrow X$ and $(X, d)$ is a metric space, is called uniform Meir-Keeler if for every $\varepsilon>0$ there exist $\eta>0$ and $\lambda>0$ such that for every $x, y \in X$ with the property that $d(x, y) \leq \varepsilon+\eta$ we have $d\left(f_{i}(x), f_{i}(y)\right)<\varepsilon-\lambda$ for every $i \in I$.

Now we present some notations concerning the shift space of an IIFS.

The set $I^{\mathbb{Z}^{+}}$is denoted by $\Lambda$ and the set $I^{\{1,2, \ldots, n\}}$ by $\Lambda_{n}$. For an IIFS $\mathcal{S}=\left(f_{i}\right)_{i \in I}, \Lambda$ is named the shift or code space of the IIFS $\mathcal{S}$. The elements of $\Lambda$ can be written as infinite words $\alpha=\alpha_{1} \alpha_{2} \alpha_{3} \cdots$ and the elements of $\Lambda_{n}$ as finite words $\alpha=\alpha_{1} \alpha_{2} \cdots \alpha_{n}$. By $\Lambda^{*}$ we denote the set of all finite words, namely $\Lambda^{*}=\bigcup_{n \in \mathbb{Z}^{+}} \Lambda_{n}$. $\Lambda$ can be seen as a metric space with the distance $d_{\Lambda}$ defined by $d_{\Lambda}(\alpha, \alpha)=0$ and for $\alpha \neq \beta, d_{\Lambda}(\alpha, \beta)=\frac{1}{2^{n}}$ where $n$ is the natural number having the property that $\alpha_{k}=\beta_{k}$ for $k<n$ and $\alpha_{n} \neq \beta_{n}$, where $\alpha=\alpha_{1} \alpha_{2} \alpha_{3} \cdots \alpha_{n} \alpha_{n+1} \cdots$ and $\beta=\beta_{1} \beta_{2} \beta_{3} \cdots \beta_{n} \beta_{n+1} \cdots$. By $\alpha \beta$ we understand the concatenation of the words $\alpha \in \Lambda^{*}$ and $\beta \in \Lambda \cup \Lambda^{*} . \tau_{i}$ denotes the function $\tau_{i}: \Lambda \rightarrow \Lambda$ defined by $\tau_{i}(\alpha)=i \alpha$ and $R$ denotes the function $R: \Lambda \rightarrow \Lambda$ defined by $R\left(\alpha_{1} \alpha_{2} \alpha_{3} \cdots\right)=\alpha_{2} \alpha_{3} \cdots$. For $\alpha \in \Lambda \cup \Lambda_{n}$ and $m \leq n$, $[\alpha]_{m}=\alpha_{1} \alpha_{2} \cdots \alpha_{m}$. By $|\alpha|$ we denote the number of letters of $\alpha$. 
We note that on $\left(\Lambda, d_{\Lambda}\right)$ the set of functions $\left(\tau_{i}\right)_{i \in I}$ forms an IIFS, consisting of injective functions with disjoint images.

On $C(\Lambda, X)$ we consider the generalized metric $d_{\infty}$ (that is, $d_{\infty}: C(\Lambda, X) \times C(\Lambda, X) \rightarrow$ $[0, \infty])$ defined by $d_{\infty}(f, g):=\sup _{\alpha \in \Lambda} d(f(\alpha), g(\alpha))$. Note that $\left(C(\Lambda, X), d_{\infty}\right)$ is a complete generalized metric space if $(X, d)$ is a complete metric space and $\left(\mathrm{CB}(\Lambda, X), d_{\infty}\right)$ is a complete metric space if $(X, d)$ is so.

Let $(X, d)$ be a metric space and $\mathcal{S}=\left(f_{i}\right)_{i \in I}$ be an IIFS on $X$. Then $f_{\alpha_{1} \alpha_{2} \cdots \alpha_{n}}:=f_{\alpha_{1}} \circ f_{\alpha_{2}} \circ$ $\cdots \circ f_{\alpha_{n}}$ and $Y_{\alpha_{1} \alpha_{2} \cdots \alpha_{n}}:=f_{\alpha_{1} \alpha_{2} \cdots \alpha_{n}}(Y)$ for a set $Y \subset X$.

Theorem 2.4 (see $[11,16])$ Let $\mathcal{S}=\left(f_{i}\right)_{i \in I}$ be an IIFS on the complete metric space $(X, d)$. If the family of functions $\left(f_{i}\right)_{i \in I}$ is uniform Meir-Keeler or $(X, d)$ is a compact metric space, $I$ is finite and all the functions $f_{i}$ are contractive, then there exists a unique set $A=A(\mathcal{S})$ such that $F_{\mathcal{S}}(A)=A$ and $h\left(F_{\mathcal{S}}^{[n]}(Y), A\right) \rightarrow 0$ for every $Y \in B(X)$. In addition, there exists a unique continuous function $\pi_{\mathcal{S}}: \Lambda \rightarrow X$ such that $\pi_{\mathcal{S}} \circ \tau_{i}=f_{i} \circ \pi_{\mathcal{S}}$ for every $i \in I, \overline{\pi_{\mathcal{S}}(\Lambda)}=A$ and $\lim _{n \rightarrow \infty} f_{[\alpha]_{n}}(x)=\pi_{\mathcal{S}}(\alpha)$ for every $x \in X$ and $\alpha \in \Lambda$. Moreover, the sequence $\left(A_{[\alpha]_{n}}\right)_{n \geq 1}$ is decreasing, $\left\{\pi_{\mathcal{S}}(\alpha)\right\}=\bigcap_{n \geq 1} \overline{A_{[\alpha]_{n}}}$ and $\lim _{n \rightarrow \infty} \sup _{\alpha \in \Lambda_{n}} d\left(A_{\alpha}\right)=0$. In the particular case when $c:=\sup _{i \in I} \operatorname{Lip}\left(f_{i}\right)<1$, we have the following estimations of the speed of convergence $h\left(F_{\mathcal{S}}^{[n]}(Y), A\right) \leq \frac{c^{n}}{1-c} h\left(F_{\mathcal{S}}(Y), Y\right)$ for every $Y \in B(X)$ and $d\left(A_{\alpha}\right) \leq c^{n} d(A)$ for every $\alpha \in \Lambda_{n}$.

Proof The existence of the set $A(\mathcal{S})$ with the properties from the theorem was proved in [11]. The function $\pi_{\mathcal{S}}$ was defined in [16] and its properties were proved there in the particular case when $c=\sup _{i \in I} \operatorname{Lip}\left(f_{i}\right)<1$. In the general case, when the family of functions $\left(f_{i}\right)_{i \in I}$ is uniform Meir-Keeler, the proof is similar (quite identical) with the exception of the fact that $\lim _{n \rightarrow \infty} \sup _{\alpha \in \Lambda_{n}} d\left(A_{\alpha}\right)=0$. We are going to prove here only this part. Since for every $\alpha \in \Lambda$ and every natural number $n$ we have $A_{[\alpha]_{n+1}} \subset A_{[\alpha]_{n}}$, it follows that $\sup _{\alpha \in \Lambda_{n}} d\left(A_{\alpha}\right) \geq \sup _{\alpha \in \Lambda_{n+1}} d\left(A_{\alpha}\right)$. Therefore the sequence $\left(\sup _{\alpha \in \Lambda_{n}} d\left(A_{\alpha}\right)\right)_{n \geq 1}$ is convergent to a number $l \geq 0$. If $l>0$, since the family of functions $\left(f_{i}\right)_{i \in I}$ is uniform Meir-Keeler, there exist $\eta>0$ and $\lambda>0$ such that for every $x, y \in X$ with the property that $d(x, y) \leq l+\eta$ we have $d\left(f_{i}(x), f_{i}(y)\right)<l-\lambda$ for all $i \in I$. Because $\lim _{n \rightarrow \infty} \sup _{\alpha \in \Lambda_{n}} d\left(A_{\alpha}\right)=l$, there exists $n_{\eta}$ such that for every $n \geq n_{\eta}$ we have $\sup _{\alpha \in \Lambda_{n}} d\left(A_{\alpha}\right)<l+\eta$. As a result, for every $n \geq n_{\eta}+1$, we have $\sup _{\alpha \in \Lambda_{n}} d\left(A_{\alpha}\right) \leq l-\lambda$. This is in contradiction with the fact that $\lim _{n \rightarrow \infty} \sup _{\alpha \in \Lambda_{n}} d\left(A_{\alpha}\right)=l$. Therefore $\lim _{n \rightarrow \infty} \sup _{\alpha \in \Lambda_{n}} d\left(A_{\alpha}\right)=0$.

Definition 2.5 The set $A=A(\mathcal{S})$ from the above theorem is called the attractor of the IIFS $\mathcal{S}=\left(f_{i}\right)_{i \in I}$ and the function $\pi_{\mathcal{S}}: \Lambda \rightarrow A$ from the above theorem is called the canonical projection between the shift space of the IIFS and its attractor.

\section{The main results}

For an IIFS $\mathcal{S}=\left(f_{i}\right)_{i \in I}$ on the metric space $(X, d)$, we consider the function $H_{\mathcal{S}}: C(\Lambda, X) \rightarrow$ $C(\Lambda, X)$ defined by

$$
H_{\mathcal{S}}(f)(\alpha)=f_{[\alpha]_{1}} \circ f \circ R(\alpha)
$$

for every $f \in C(\Lambda, X)$ and $\alpha \in \Lambda$.

We remark that $H_{\mathcal{S}}$ is well defined. Indeed $\left.H_{\mathcal{S}}(f)\right|_{\Lambda_{i}}$ is continuous for every $i \in I$ since $f_{i}, f$ and $R$ are continuous functions. As the sets $\Lambda_{i}, i \in I$, are disjoint, open and closed, it follows that $H_{\mathcal{S}}(f)$ is a continuous function. 
We also note that $H_{\mathcal{S}}(\mathrm{CB}(\Lambda, X)) \subset \mathrm{CB}(\Lambda, X)$. Indeed, for a function $f \in \mathrm{CB}(\Lambda, X), f(\Lambda)$ is bounded and $H_{\mathcal{S}}(f)(\Lambda)=H_{\mathcal{S}}(f)\left(\bigcup_{i \in I} \Lambda_{i}\right)=\bigcup_{i \in I} f_{i}(f(\Lambda))$. Taking into account that the family of functions $\left(f_{i}\right)_{i \in I}$ is bounded, we obtain the desired result.

Proposition 3.1 Let $\mathcal{S}=\left(f_{i}\right)_{i \in I}$ be an IIFS on the metric space $(X, d)$. Then:

(i) If the family of functions $\left(f_{i}\right)_{i \in I}$ is equal uniform continuous, then $H_{\mathcal{S}}$ is continuous. In particular, if $I$ is finite, then $H_{\mathcal{S}}$ is continuous.

(ii) We have $\operatorname{Lip}\left(H_{\mathcal{S}}\right) \leq \sup _{i \in I} \operatorname{Lip}\left(f_{i}\right)$. In particular, if $\sup _{i \in I} \operatorname{Lip}\left(f_{i}\right)<1$, then $H_{\mathcal{S}}$ is a contraction. If $f_{i}$ are contractions and I is finite, then $\sup _{i \in I} \operatorname{Lip}\left(f_{i}\right)<1$.

(iii) If all $f_{i}$ are $\phi$-contractions, where $\phi$ is a comparison function, then $H_{\mathcal{S}}$ is a $\phi$-contraction.

(iv) If the family of functions $\left(f_{i}\right)_{i \in I}$ is uniform Meir-Keeler, then $H_{\mathcal{S}}$ is Meir-Keeler.

(v) If I is finite and all the functions $f_{i}$ are Meir-Keeler, then $H_{\mathcal{S}}$ is Meir-Keeler.

(vi) If I is finite and all the functions $f_{i}$ are contractive, then $H_{\mathcal{S}}$ is contractive.

Proof (i) Let $\varepsilon>0$ be fixed. Then there exists $\delta_{\varepsilon}>0$ such that $d\left(f_{i}(x), f_{i}(y)\right)<\varepsilon$ for every $x, y \in X$ such that $d(x, y)<\delta_{\varepsilon}$ and $i \in I$. We suppose that $g_{n} \rightarrow g$ in $\left(C(\Lambda, X), d_{\infty}\right)$. Consequently, there exists $n_{\varepsilon}$ such that $d_{\infty}\left(g_{n}, g\right)<\delta_{\varepsilon}$ for every $n \geq n_{\varepsilon}$. Then $d\left(H_{\mathcal{S}}\left(g_{n}\right)(\alpha)\right.$, $\left.H_{\mathcal{S}}(g)(\alpha)\right)=d\left(f_{[\alpha]_{1}} \circ g_{n} \circ R(\alpha), f_{[\alpha]_{1}} \circ g \circ R(\alpha)\right)<\varepsilon$. It follows that $d_{\infty}\left(H_{\mathcal{S}}\left(g_{n}\right), H_{\mathcal{S}}(g)\right) \leq \varepsilon$ for every $n \geq n_{\varepsilon}$.

(ii) We have

$$
\begin{aligned}
d_{\infty}\left(H_{\mathcal{S}}(f), H_{\mathcal{S}}(g)\right) & =\sup _{\alpha \in \Lambda} d\left(H_{\mathcal{S}}(f)(\alpha), H_{\mathcal{S}}(g)(\alpha)\right) \\
& =\sup _{\alpha \in \Lambda} d\left(f_{[\alpha]_{1}} \circ f \circ R(\alpha), f_{[\alpha]_{1}} \circ g \circ R(\alpha)\right) \\
& \leq \sup _{\alpha \in \Lambda} \operatorname{Lip}\left(f_{[\alpha]_{1}}\right) d(f \circ R(\alpha), g \circ R(\alpha)) \\
& \leq \sup _{\alpha \in \Lambda}\left(\sup _{i \in I} \operatorname{Lip}\left(f_{i}\right)\right) d(f \circ R(\alpha), g \circ R(\alpha)) \\
& =\left(\sup _{i \in I} \operatorname{Lip}\left(f_{i}\right)\right)\left(\sup _{\alpha \in \Lambda} d(f \circ R(\alpha), g \circ R(\alpha))\right) \\
& =\left(\sup _{i \in I} \operatorname{Lip}\left(f_{i}\right)\right) \sup _{\alpha \in \Lambda} d(f(\alpha), g(\alpha))=\left(\sup _{i \in I} \operatorname{Lip}\left(f_{i}\right)\right) d_{\infty}(f, g) .
\end{aligned}
$$

(iii) We have

$$
\begin{aligned}
d_{\infty}\left(H_{\mathcal{S}}(f), H_{\mathcal{S}}(g)\right) & =\sup _{\alpha \in \Lambda} d\left(H_{\mathcal{S}}(f)(\alpha), H_{\mathcal{S}}(g)(\alpha)\right) \\
& =\sup _{\alpha \in \Lambda} d\left(f_{[\alpha]_{1}} \circ f \circ R(\alpha), f_{[\alpha]_{1}} \circ g \circ R(\alpha)\right) \\
& \leq \sup _{\alpha \in \Lambda} \phi(d(f \circ R(\alpha), g \circ R(\alpha))) \\
& \leq \phi\left(\sup _{\alpha \in \Lambda} d(f \circ R(\alpha), g \circ R(\alpha))\right)=\phi\left(d_{\infty}(f, g)\right) .
\end{aligned}
$$

(iv) Let $\varepsilon>0$ be fixed. Then there exist $\eta>0$ and $\lambda>0$ such that for every $x, y \in X$ with the property that $d(x, y)<\varepsilon+\eta$ we have $d\left(f_{i}(x), f_{i}(y)\right)<\varepsilon-\lambda$. We suppose that $d_{\infty}(f, g)<$ $\varepsilon+\eta$. Then $d(f(\alpha), g(\alpha))<\varepsilon+\eta$ for every $\alpha \in \Lambda$. In particular $d(f \circ R(\alpha), g \circ R(\alpha))<\varepsilon+\eta$ 
for every $\alpha \in \Lambda$. Therefore $d\left(f_{[\alpha]_{1}} \circ f \circ R(\alpha), f_{[\alpha]_{1}} \circ g \circ R(\alpha)\right)=d\left(H_{\mathcal{S}}(f)(\alpha), H_{\mathcal{S}}(g)(\alpha)\right)<\varepsilon-\lambda$ for every $i \in I$. It follows that $d_{\infty}\left(H_{\mathcal{S}}(f), H_{\mathcal{S}}(g)\right)=\sup _{\alpha \in \Lambda} d\left(H_{\mathcal{S}}(f)(\alpha), H_{\mathcal{S}}(g)(\alpha)\right) \leq \varepsilon-\lambda<$ $\varepsilon-\lambda / 2$, which gives the desired result.

(v) Let $\varepsilon>0$ be fixed. Then there exists $\eta>0$ such that for every $x, y \in X$ with the property that $d(x, y)<\varepsilon+\eta$ we have $d\left(f_{i}(x), f_{i}(y)\right)<\varepsilon$ for every $i \in I$. We suppose that $d_{\infty}(f, g)<\varepsilon+\eta$. Then $d(f(\alpha), g(\alpha))<\varepsilon+\eta$ for every $\alpha \in \Lambda$ and $d\left(f_{\alpha_{1}} \circ f \circ R(\alpha), f_{\alpha_{1}} \circ g \circ R(\alpha)\right)<\varepsilon$ for every $\alpha \in \Lambda$ and $i \in I$. Since

$$
d_{\infty}\left(H_{\mathcal{S}}(f), H_{\mathcal{S}}(g)\right)=\sup _{\alpha \in \Lambda} d\left(f_{[\alpha]_{1}} \circ f \circ R(\alpha), f_{[\alpha]_{1}} \circ g \circ R(\alpha)\right)
$$

$I$ is finite and $\Lambda$ is compact, there exists $\beta \in \Lambda$ such that

$$
d_{\infty}\left(H_{\mathcal{S}}(f), H_{\mathcal{S}}(g)\right)=d\left(f_{[\beta]_{1}} \circ f \circ R(\beta), f_{[\beta]_{1}} \circ g \circ R(\beta)\right) .
$$

It follows that $d_{\infty}\left(H_{\mathcal{S}}(f), H_{\mathcal{S}}(g)\right)<\varepsilon$.

(vi) We have $d_{\infty}\left(H_{\mathcal{S}}(f), H_{\mathcal{S}}(g)\right)=\sup _{\alpha \in \Lambda} d\left(f_{[\alpha]_{1}} \circ f \circ R(\alpha), f_{[\alpha]_{1}} \circ g \circ R(\alpha)\right)$. Since $I$ is finite and $\Lambda$ is compact, there exists $\beta \in \Lambda$ such that $d_{\infty}\left(H_{\mathcal{S}}(f), H_{\mathcal{S}}(g)\right)=d\left(f_{[\beta]_{1}} \circ f \circ R(\beta), f_{[\beta]_{1}} \circ\right.$ $g \circ R(\beta))$. Then

$$
\begin{aligned}
d_{\infty}\left(H_{\mathcal{S}}(f), H_{\mathcal{S}}(g)\right) & =d\left(f_{[\beta]_{1}} \circ f \circ R(\beta), f_{[\beta]_{1}} \circ g \circ R(\beta)\right) \\
& <d(f \circ R(\beta), g \circ R(\beta)) \leq d_{\infty}(f, g) .
\end{aligned}
$$

Note that if all the functions $f_{i}$ are $\phi$-contractions for a comparison function $\phi$, then the family of functions $\left(f_{i}\right)_{i \in I}$ is uniform Meir-Keeler. We also remark that if $\sup _{i \in I} \operatorname{Lip}\left(f_{i}\right)<1$, then there exists a comparison function $\phi$ such that all $f_{i}$ are $\phi$-contractions, namely $\phi(r)=$ $r \sup _{i \in I} \operatorname{Lip}\left(f_{i}\right)$.

Theorem 3.2 Let $\mathcal{S}=\left(f_{i}\right)_{i \in I}$ be an IIFS on the complete metric space $(X, d)$. Then there exists a unique fixed point $\pi_{0}$ of $H_{\mathcal{S}}$ and $d_{\infty}\left(H_{\mathcal{S}}^{[n]}(f), \pi_{0}\right) \rightarrow 0$ for every $f \in \mathrm{CB}(\Lambda, X)$ provided that one of the following two conditions is fulfilled:

(i) the family of functions $\left(f_{i}\right)_{i \in I}$ is uniform Meir-Keeler;

(ii) $(X, d)$ is a compact metric space, $I$ is finite and all $f_{i}$ are contractive.

Moreover, $\pi_{0} \circ \tau_{i}=f_{i} \circ \pi_{0}$ for every $i \in I$; and consequently $\pi_{0}=\pi_{\mathcal{S}}$. In the particular case when $c:=\sup _{i \in I} \operatorname{Lip}\left(f_{i}\right)<1$, we have the following estimations of the speed of convergence:

$$
d_{\infty}\left(H_{\mathcal{S}}^{[n]}(f), \pi_{0}\right) \leq \frac{c^{n}}{1-c} d_{\infty}\left(H_{\mathcal{S}}(f), f\right) .
$$

Proof In the first case (the family of functions $\left(f_{i}\right)_{i \in I}$ is uniform Meir-Keeler) the existence of $\pi_{0}$ results from Theorem 2.2, Proposition 3.1 and the fact that the space $\left(\mathrm{CB}(\Lambda, X), d_{\infty}\right)$ is complete. For the second case $\left((X, d)\right.$ is a compact metric space, $I$ is finite and all $f_{i}$ are contractive), we remark that $f_{i}$ are $\phi$-contractions for the comparison function $\phi(r)=$ $\sup \left\{d\left(f_{i}(x), f_{i}(y)\right): i \in I\right.$ and $\left.d(x, y) \leq r\right\}$, and so this case results from the previous one.

From the fact that $H_{\mathcal{S}}\left(\pi_{0}\right)=\pi_{0}$ we infer that $\pi_{0} \circ \tau_{i}(\alpha)=\pi_{0}(i \alpha)=H_{\mathcal{S}} \circ \pi_{0}(i \alpha)=f_{i}\left(\pi_{0}(\alpha)\right)$ for every $\alpha \in \Lambda$ and $i \in I$, i.e., $\pi_{0} \circ \tau_{i}=f_{i} \circ \pi_{0}$ for every $i \in I$.

For $\alpha, \beta \in \Lambda$, we have $[\alpha]_{n} \beta \rightarrow \alpha$ in $\left(\Lambda, d_{\Lambda}\right)$; and consequently $\pi_{0}\left([\alpha]_{n} \beta\right) \rightarrow \pi_{0}(\alpha)$. But $\pi_{0}\left([\alpha]_{n} \beta\right)=\pi_{0} \circ \tau_{[\alpha]_{n}}(\beta)=f_{[\alpha]_{n}} \circ \pi_{0}(\beta)$ and, from Theorem 2.4, we infer that $f_{[\alpha]_{n}} \circ \pi_{0}(\beta) \rightarrow$ 
$\pi_{\mathcal{S}}(\alpha)$. Therefore $\pi_{0}=\pi_{\mathcal{S}}$. The estimation of the speed of convergence comes from the Banach contraction principle.

Remarks 3.3 (i) We suppose that $(X, d)$ is a complete metric space and $\mathcal{S}=\left(f_{i}\right)_{i \in I}$ is an IFS ( $I$ is a finite set). Then we have

$$
H_{\mathcal{S}}(f)(\Lambda)=H_{\mathcal{S}}\left(f\left(\bigcup_{i \in I} \Lambda_{i}\right)\right)=\bigcup_{i \in I} H_{\mathcal{S}}\left(f\left(\Lambda_{i}\right)\right)=\bigcup_{i \in I} f_{i}(f(\Lambda))
$$

for every $f \in C(\Lambda, X)$.

Since $I$ is finite, it follows that $\Lambda$ and $f(\Lambda)$ are compact, and so

$$
H_{\mathcal{S}}(f)(\Lambda)=\bigcup_{i \in I} f_{i}(f(\Lambda))=\bigcup_{i \in I} \overline{f_{i}(f(\Lambda))}=\overline{\bigcup_{i \in I} f_{i}(f(\Lambda))}=F_{\mathcal{S}}(f(\Lambda)) .
$$

By induction one can prove that

$$
H_{\mathcal{S}}^{[n]}(f)(\Lambda)=F_{\mathcal{S}}^{[n]}(f(\Lambda))
$$

for every $n \in \mathbb{N}$.

(ii) If in addition $f_{i}$ are Meir-Keeler, then $d_{\infty}\left(H_{\mathcal{S}}^{[n]}(f), \pi_{0}\right) \rightarrow 0$, which implies that $h\left(H_{\mathcal{S}}^{[n]}(f)(\Lambda), \pi_{0}(\Lambda)\right) \rightarrow 0$. From the first part of the remark we obtain $h\left(F_{\mathcal{S}}^{[n]}(f(\Lambda))\right.$, $\left.\pi_{0}(\Lambda)\right) \rightarrow 0$. Since $H_{\mathcal{S}}\left(\pi_{0}\right)=\pi_{0}$, we have

$$
F_{\mathcal{S}}\left(\pi_{0}(\Lambda)\right)=\pi_{0}(\Lambda)
$$

(iii) Let $M \in K(X)$. We suppose that $I$ has at least two elements. In this case $\Lambda$ is homeomorphic with the Cantor set. It is known that there exists a continuous function $f_{M}$ from $\Lambda$ onto $M$. We have proved that $F_{\mathcal{S}}\left(\pi_{0}(\Lambda)\right)=\pi_{0}(\Lambda)$ and that $h\left(F_{\mathcal{S}}^{[n]}(M), \pi_{0}(\Lambda)\right)=$ $h\left(F_{\mathcal{S}}^{[n]}\left(f_{M}(\Lambda)\right), \pi_{0}(\Lambda)\right) \rightarrow 0$ for every $M \in K(X)$. If $F_{\mathcal{S}}(M)=M$ for a set $M \in K(X)$, then $h\left(M, \pi_{0}(\Lambda)\right)=h\left(F_{\mathcal{S}}^{[n]}(M), \pi_{0}(\Lambda)\right) \rightarrow 0$. It follows that $M=\pi_{0}(\Lambda)$.

In this way, the first part of Theorem 3.2 implies the first part of Theorem 2.4.

(iv) A similar result could be obtained when $I$ is an infinite set with the differences that $\overline{H_{\mathcal{S}}(f)(\Lambda)}=F_{\mathcal{S}}(f(\Lambda)), \overline{H_{\mathcal{S}}^{[n]}(f)(\Lambda)}=F_{\mathcal{S}}^{[n]}(f(\Lambda))$, the attractor is $\overline{\pi_{0}(\Lambda)}$ instead of $\pi_{0}(\Lambda)$ and the convergence is obtained for sets from $B_{I}(X)$.

\section{The case of $\varepsilon$-chainable spaces}

In this section we intend to obtain similar results as those from Theorem 3.2 when we know that the contractive conditions are satisfied only for closed enough points. Since the Meir-Keeler is the most general contractive condition (from the list from Section 1) which assures the existence of the fixed point on an arbitrary complete metric space, we will study only two cases, namely:

(i) $\varepsilon$-Meir-Keeler functions on arbitrary complete metric spaces, and

(ii) $\varepsilon$-contractive functions on compact metric spaces (see Definition 4.8).

The corresponding fixed point result of Theorem 2.2 (Theorem 4.9) requires for the basic space to satisfy certain conditions of $\varepsilon$-chainability (see Definition 4.1 ), so we need to have a similar property. In fact we need this property for the space $\mathrm{CB}(\Lambda, X)$. It is a natural 
question if $\mathrm{CB}(\Lambda, X)$ inherits this property from $X$. This happens if $I$ is finite but does not always happen if $I$ is infinite (see Examples 4.3, 4.4 and 4.5). A similar fact happens for $K(X)$ and $B(X) . K(X)$ is always $\varepsilon$-chainable when $X$ is so, but $B(X)$ is not (see [11]). Therefore we will use the notations of $\varepsilon$-chainable and uniform $\varepsilon$-chainable metric spaces. In Proposition 4.2 it is proved that $\mathrm{CB}(\Lambda, X)$ is $\varepsilon$-chainable if $I$ is finite and in Proposition 4.7 the same conclusion is obtained for $I$ infinite. The case when $I$ is a finite set is straightforward, but the case when $I$ is infinite, although it has a similar idea, requires a preparatory result, namely Lemma 4.6.

Definition 4.1 Let $\varepsilon>0$. A metric space $(X, d)$ is called $\varepsilon$-chainable if for every $x, y \in X$ there exists a chain $x_{0}=x, x_{1}, \ldots, x_{n}=y$ such that $d\left(x_{k}, x_{k+1}\right)<\varepsilon$ for every $k \in\{0,1, \ldots, n-1\}$, and it is called uniform $\varepsilon$-chainable if for every $M>0$ there exists a natural number $n_{M}$ such that for every $x, y \in X$ with the property that $d(x, y)<M$ there exists a chain $x_{0}=$ $x, x_{1}, \ldots, x_{n}=y$ such that $d\left(x_{k}, x_{k+1}\right)<\varepsilon$ for every $k \in\{0,1, \ldots, n-1\}$ and $n \leq n_{M}$. A chain $x_{0}, x_{1}, \ldots, x_{n}$ such that $d\left(x_{k}, x_{k+1}\right)<\varepsilon$ for every $k \in\{0,1, \ldots, n-1\}$ is called an $\varepsilon$-chain.

Proposition 4.2 If the metric space $(X, d)$ is $\varepsilon$-chainable and I is finite, then the metric space $C(\Lambda, X)=\mathrm{CB}(\Lambda, X)$ is $\varepsilon$-chainable.

Proof Let $f, g \in C(\Lambda, X)$. Since $\Lambda$ is a compact set, it follows that $f$ and $g$ are uniform continuous functions. Hence there is a natural number $m$ such that for every $\alpha \in \Lambda_{m}$ and $\beta, \gamma \in \Lambda$ we have $d(f(\alpha \beta), f(\alpha \gamma))<\varepsilon$ and $d(g(\alpha \beta), g(\alpha \gamma))<\varepsilon$. Let $f_{1}$ and $g_{1}$ be defined by $\left.f_{1}\right|_{\Lambda_{\alpha}}=f(\alpha \beta)$ for every $\alpha \in \Lambda_{m}$ and $\left.g_{1}\right|_{\Lambda_{\alpha}}=g(\alpha \beta)$ for every $\alpha \in \Lambda_{m}$, where $\beta$ is a fixed element of $\Lambda$. We have $d_{\infty}\left(f, f_{1}\right)<\varepsilon$ and $d_{\infty}\left(g, g_{1}\right)<\varepsilon$. Since $(X, d)$ is $\varepsilon$-chainable, for every $\alpha \in \Lambda_{m}$ there exists an $\varepsilon$-chain $x_{0}^{\alpha}=f(\alpha \beta)=f_{1}(\alpha \beta), x_{1}^{\alpha}, \ldots, x_{n^{\alpha}}^{\alpha}=g(\alpha \beta)=g_{1}(\alpha \beta)$. Because $I$ is finite, we can suppose that $n^{\alpha}=n$ for every $\alpha \in \Lambda_{m}$. We consider the functions $f_{k} \in C(\Lambda, X)$ defined by $\left.f_{k}\right|_{\Lambda_{\alpha}}=x_{k}^{\alpha}$ for every $\alpha \in \Lambda_{m}$ and remark that $f_{n}=g_{1}$ and $f, f_{1}, f_{2}, \ldots, f_{n}, g$ is an $\varepsilon$-chain between $f$ and $g$.

We give some examples which show that $\mathrm{CB}(\Lambda, X)$ could not be an $\varepsilon$-chainable metric space for every $\varepsilon>0$, although $X$ is $\varepsilon$-chainable for every $\varepsilon>0$. As usual, $l_{2}$ denotes the Hilbert space of sequences of real numbers $x=\left(x_{n}\right)_{n \geq 1}$ such that $\sum_{n=1}^{\infty} x_{n}^{2}<\infty$ endowed with the norm $\|x\|_{2}=\sqrt{\sum_{n=1}^{\infty} x_{n}^{2}}$. We denote by $e_{n}$ the element $\left(\delta_{m}^{n}\right)_{m \geq 1}$, where $\delta_{m}^{n}=\left\{\begin{array}{ll}1 & \text { if } n=m, \\ 0 & \text { if } n \neq m\end{array}\right.$ and by $d_{2}$ the distance associated with the norm $\|\circ\|_{2}$. For $x, y \in l_{2},[x, y]$ is the set $\{t x+(1-t) y: t \in[0,1]\}$. Let $X=\bigcup_{n \geq 1}\left[e_{n}, e_{n+1}\right] \subset l_{2}$.

Example 4.3 With the above notations the metric space $\left(X, d_{2}\right)$ is an $\varepsilon$-chainable metric space for every $\varepsilon>0$, but $\mathrm{CB}(\Lambda, X)$ is not an $\varepsilon$-chainable metric space for every $\varepsilon \in\left(0, \frac{1}{\sqrt{2}}\right)$.

Indeed, since $\left(X, d_{2}\right)$ is an arcwise connected space it is also an $\varepsilon$-chainable metric space. Let us consider the functions $f, g \in \mathrm{CB}(\Lambda, X)$ defined by $f(\alpha)=e_{1}$ and $g(n \alpha)=e_{n}$ for every $\alpha \in \Lambda$ and $n \in \mathbb{Z}^{+}$, where $\Lambda=\Lambda\left(\mathbb{Z}^{+}\right)$and $\mathbb{Z}^{+}$denotes the set of strict positive natural numbers. If there exists an $\varepsilon$-chain in $\mathrm{CB}(\Lambda, X), f_{0}=f, f_{1}, \ldots, f_{n}=g$, for an $\varepsilon \in\left(0, \frac{1}{\sqrt{2}}\right)$, then for every $m \in \mathbb{Z}^{+}, f_{0}(m \alpha)=f(m \alpha)=e_{1}, f_{1}(m \alpha), \ldots, f_{n}(m \alpha)=g(m \alpha)=e_{m}$ is an $\varepsilon$-chain with $n+1$ terms between $e_{1}$ and $e_{m}$ for every $\alpha \in \Lambda$ and $m \in \mathbb{Z}^{+}$. Since for every $l, k \in \mathbb{Z}^{+}$such that $l+1<m$ we have

$$
\delta\left(\left[e_{l}, e_{l+1}\right],\left[e_{k}, e_{k+1}\right]\right)=\inf _{x \in\left[e_{l}, e_{l+1}\right], y \in\left[e_{k}, e_{k+1}\right]} d(x, y) \geq \frac{1}{\sqrt{2}},
$$


it follows that an $\varepsilon$-chain with $\varepsilon \in\left(0, \frac{1}{\sqrt{2}}\right)$ between $e_{1}$ and $e_{m}$ should have at least $m-1$ elements for every natural number $m$ and this leads to a contradiction.

Example 4.4 We consider on the set of real numbers the distance $d_{m}$ defined by $d_{m}(x, y)=$ $\min \{|x-y|, m\}$, where $m>0$. In a similar way as above, it can be seen that $\left(\mathbb{R}, d_{m}\right)$ is an $\varepsilon$-chainable metric space for every $\varepsilon>0$, but $\mathrm{CB}\left(\Lambda\left(\mathbb{Z}^{+}\right),\left(\mathbb{R}, d_{m}\right)\right)$ is not an $\varepsilon$-chainable metric space for every $\varepsilon \in(0, m)$.

Moreover, one can find an $\varepsilon$-chainable metric space for every $\varepsilon>0,(X, d)$, such that $\mathrm{CB}\left(\Lambda\left(\mathbb{Z}^{+}\right),(X, d)\right)$ is not an $\varepsilon$-chainable metric space for every $\varepsilon>0$ as we can see from the following example.

Example 4.5 With the notations from Example 4.3 we consider the set $Y=\mathbb{R} e_{1} \cup$ $\left(\bigcup_{m, n \geq 1}\left[m^{2} e_{n}, m^{2} e_{n+1}\right]\right) \subset l_{2}$. The metric space $\left(Y, d_{2}\right)$ is an $\varepsilon$-chainable metric space for every $\varepsilon>0$, but $\mathrm{CB}(\Lambda, Y)$ is not an $\varepsilon$-chainable metric space for every $\varepsilon>0$, where $\Lambda=\Lambda\left(\mathbb{Z}^{+}\right)$.

For the proof of the fact that $\mathrm{CB}(\Lambda, X)$ is an $\varepsilon$-chainable metric space when $(X, d)$ is uniform $\varepsilon$-chainable, we need the following result.

Lemma 4.6 Let I be a set and $f, g \in C(\Lambda, X)$. Then there exists a set $\Gamma \subset \Lambda^{*}$ such that for every $\alpha, \beta \in \Gamma, \alpha \neq \beta$, we have:

(i) $\Lambda_{\alpha} \cap \Lambda_{\beta}=\emptyset$;

(ii) $\bigcup_{\alpha \in \Gamma} \Lambda_{\alpha}=\Lambda$;

(iii) $\max \left\{\sup _{\beta, \gamma \in \Lambda, \alpha \in \Gamma} d(f(\alpha \beta), f(\alpha \gamma)), \sup _{\beta, \gamma \in \Lambda, \alpha \in \Gamma} d(g(\alpha \beta), g(\alpha \gamma))\right\}<\varepsilon$.

Proof We define by induction, for $n \geq 1$, the sets $\Gamma_{n}$ in the following way:

$$
\Gamma_{1}=\left\{\Lambda_{i}: i \in I, \max \left\{\sup _{\beta, \gamma \in \Lambda} d(f(i \beta), f(i \gamma)) \sup _{\beta, \gamma \in \Lambda} d(g(i \beta), g(i \gamma))\right\}<\varepsilon\right\} .
$$

We suppose that we have defined $\Gamma_{k}$ for $k \in\{1,2, \ldots, n\}$. Then

$$
\begin{aligned}
\Gamma_{n+1}= & \left\{\Lambda_{\alpha}: \alpha \in \Lambda_{n+1}, \Lambda_{\alpha} \cap \Lambda_{\beta}=\emptyset \text { for every } \beta \in \bigcup_{i=1}^{n} \Gamma_{i}\right. \text { and } \\
& \left.\max \left\{\sup _{\beta, \gamma \in \Lambda} d(f(\alpha \beta), f(\alpha \gamma)), \sup _{\beta, \gamma \in \Lambda} d(g(\alpha \beta), g(\alpha \gamma))\right\}<\varepsilon\right\} .
\end{aligned}
$$

We remark that $\Lambda_{\alpha} \cap \Lambda_{\beta}=\emptyset$ for every $\alpha, \beta \in \Lambda_{n}, \alpha \neq \beta$.

Let $\Gamma=\bigcup_{n \geq 1} \Gamma_{n}$. It is clear that for every $\alpha, \beta \in \Gamma, \alpha \neq \beta$, we have

$$
\Lambda_{\alpha} \cap \Lambda_{\beta}=\varnothing
$$

and

$$
\max \left\{\sup _{\beta, \gamma \in \Lambda, \alpha \in \Gamma} d(f(\alpha \beta), f(\alpha \gamma)), \sup _{\beta, \gamma \in \Lambda, \alpha \in \Gamma} d(g(\alpha \beta), g(\alpha \gamma))\right\}<\varepsilon
$$


for every $\alpha \in \Gamma$. We suppose by absurdum that $\bigcup_{\alpha \in \Gamma} \Lambda_{\alpha} \neq \Lambda$. Then there exists $x \in \Lambda \backslash$ $\bigcup_{\alpha \in \Gamma} \Lambda_{\alpha}$. Since $f$ and $g$ are continuous functions, there exists $\alpha_{0}$ with a minimal number of letters such that $x \in \Lambda_{\alpha_{0}}$ and

$$
\max \left\{\sup _{\beta, \gamma \in \Lambda} d\left(f\left(\alpha_{0} \beta\right), f\left(\alpha_{0} \gamma\right)\right), \sup _{\beta, \gamma \in \Lambda} d\left(g\left(\alpha_{0} \beta\right), g\left(\alpha_{0} \gamma\right)\right)\right\}<\varepsilon
$$

Let $n_{0}$ be the number of letters from $\alpha_{0}$. We will prove that $\alpha_{0} \in \Gamma_{n_{0}}$. If not, there exists $\alpha_{1} \in \Gamma_{k}$ such that $k<n_{0}$ and $\Lambda_{\alpha_{1}} \cap \Lambda_{\alpha_{0}} \neq \emptyset$. It results in $\left[\alpha_{0}\right]_{\left|\alpha_{1}\right|}=\alpha_{1}$, which contradicts the fact that $\alpha_{0}$ is a word with a minimal number of letters such that

$$
\max \left\{\sup _{\beta, \gamma \in \Lambda} d\left(f\left(\alpha_{0} \beta\right), f\left(\alpha_{0} \gamma\right)\right), \sup _{\beta, \gamma \in \Lambda} d\left(g\left(\alpha_{0} \beta\right), g\left(\alpha_{0} \gamma\right)\right)\right\}<\varepsilon
$$

Proposition 4.7 If $(X, d)$ is uniform $\varepsilon$-chainable, then $\mathrm{CB}(\Lambda, X)$ is $\varepsilon^{\prime}$-chainable for every $\varepsilon^{\prime}>\varepsilon$.

Proof Let $f, g \in \mathrm{CB}(\Lambda, X)$. Then there exists a set $\Gamma \subset \Lambda^{*}$ such that for every $\alpha, \beta \in \Gamma$, $\alpha \neq \beta$, we have $\Lambda_{\alpha} \cap \Lambda_{\beta}=\emptyset, \bigcup_{\alpha \in \Gamma} \Lambda_{\alpha}=\Lambda$ and

$$
\max \left\{\sup _{\beta, \gamma \in \Lambda, \alpha \in \Gamma} d(f(\alpha \beta), f(\alpha \gamma)), \sup _{\beta, \gamma \in \Lambda, \alpha \in \Gamma} d(g(\alpha \beta), g(\alpha \gamma))\right\}<\varepsilon / 2 .
$$

Let $f_{1}$ and $g_{1}$ be defined by $\left.f_{1}\right|_{\Lambda_{\alpha}}=f(\alpha \beta)$ for every $\alpha \in \Gamma$ and $\left.g_{1}\right|_{\Lambda_{\alpha}}=g(\alpha \beta)$ for every $\alpha \in \Gamma$, where $\beta$ is a fixed element of $\Lambda$. We have $d_{\infty}\left(f, f_{1}\right) \leq \varepsilon / 2<\varepsilon$ and $d_{\infty}\left(g, g_{1}\right) \leq \varepsilon / 2<\varepsilon$. We remark that $f_{1}, g_{1} \in \mathrm{CB}(\Lambda, X)$. Since $(X, d)$ is $\varepsilon$-chainable for every $\alpha \in \Gamma$, there exists an $\varepsilon$-chain $x_{0}^{\alpha}=f(\alpha \beta)=f_{1}(\alpha \beta), x_{1}^{\alpha}, \ldots, x_{n^{\alpha}}^{\alpha}=g(\alpha \beta)=g_{1}(\alpha \beta)$. Because $(X, d)$ is uniform $\varepsilon$-chainable, we can suppose that there exists $n$ such that $n^{\alpha}=n$ for every $\alpha \in \Gamma$. We consider the functions $f_{k} \in C(\Lambda, X)$ defined by $\left.f_{k}\right|_{\Lambda_{\alpha}}=x_{k}^{\alpha}$ for every $\alpha \in \Gamma$. It can be seen that $f_{n}=g_{1}, f_{k} \in \mathrm{CB}(\Lambda, X)$ and $d\left(f_{k}, f_{k+1}\right) \leq \varepsilon$. Then $f, f_{1}, f_{2}, \ldots, f_{n}, g$ is an $\varepsilon^{\prime}$-chain between $f$ and $g$ for every $\varepsilon^{\prime}>\varepsilon$.

As in Section 2 we consider some $\varepsilon$-contractive conditions which we will use in the sequel.

Definition 4.8 Let $(X, d)$ be a metric space, a function $f: X \rightarrow X$ and $\varepsilon>0$.

(i) $f$ is an $(\varepsilon, \phi)$-contraction if $d(f(x), f(y)) \leq \phi(d(x, y))$ for every $x, y \in X$ such that $d(x, y)<\varepsilon$, where $\phi$ is a comparison function.

(ii) We say that $f$ is an $\varepsilon$-Meir-Keeler function if for every $\varepsilon^{\prime} \in(0, \varepsilon)$ there exists $\eta>0$ such that for every $x, y \in X$ with the property that $d(x, y) \leq \varepsilon^{\prime}+\eta$ we have $d(f(x), f(y))<\varepsilon^{\prime}$. A family of functions $\left(f_{i}\right)_{i \in I}$, where $f_{i}: X \rightarrow X$, is called uniform $\varepsilon$-Meir-Keeler if for every $\varepsilon^{\prime} \in(0, \varepsilon)$ there exist $\eta>0$ and $\lambda>0$ such that for every $x, y \in X$ with the property that $d(x, y) \leq \varepsilon^{\prime}+\eta$ we have $d\left(f_{i}(x), f_{i}(y)\right)<\varepsilon^{\prime}-\lambda$ for every $i \in I$.

(iii) $f$ is called $\varepsilon$-contractive if $d(f(x), f(y))<d(x, y)$ for every $x, y \in X$ such that $d(x, y)<\varepsilon$ and $x \neq y$.

Theorem 2.2 has the following version for $\varepsilon$-Meir-Keeler functions. 
Theorem 4.9 (see [11]) Let $(X, d)$ be a complete metric space, a function $f: X \rightarrow X$ and $\varepsilon>0$. Iff is $\varepsilon$-Meir-Keeler and $X$ is $\varepsilon$-chainable for some $\varepsilon$, then there exists a unique fixed point $x_{0}$ of $f$ and the sequence $\left(f^{[n]}\left(x_{0}\right)\right)_{n}$ is convergent to $x_{0}$ for every $x \in X$. If $(X, d)$ is compact and $\varepsilon$-chainable and $f$ is $\varepsilon$-contractive, then there exists a unique fixed point $x_{0}$ off and the sequence $\left(f^{[n]}\left(x_{0}\right)\right)_{n}$ is convergent to $x_{0}$ for every $x \in X$.

We present now the version of Theorem 2.4 concerning the existence of the attractor and the canonical projection for an IIFS for the case of $\varepsilon$-chainable metric spaces.

Theorem 4.10 (see $[11,16]$ ) Let $\mathcal{S}=\left(f_{i}\right)_{i \in I}$ be an IIFS on the complete metric space $(X, d)$ and $0<\varepsilon^{\prime}<\varepsilon$. Then there exists a unique set $A=A(\mathcal{S})$ such that $F_{\mathcal{S}}(A)=A$ and $h\left(F_{\mathcal{S}}^{[n]}(Y), A\right) \rightarrow 0$ for every $Y \in B(X)$ provided that one of the following two conditions is fulfilled:

(i) the family of functions $\left(f_{i}\right)_{i \in I}$ is $\varepsilon$-uniform Meir-Keeler and $(X, d)$ is uniform $\varepsilon^{\prime}$-chainable;

(ii) $(X, d)$ is a compact $\varepsilon^{\prime}$-chainable metric space, $I$ is finite and all the functions $f_{i}$ are $\varepsilon$-contractive.

In addition, there exists a unique continuous function $\pi_{\mathcal{S}}: \Lambda \rightarrow X$ such that $\pi_{\mathcal{S}} \circ$ $\tau_{i}=f_{i} \circ \pi_{\mathcal{S}}$ for every $i \in I, \overline{\pi_{\mathcal{S}}(\Lambda)}=A$ and $\lim _{n \rightarrow \infty} f_{[\alpha]_{n}}(x)=\pi_{\mathcal{S}}(\alpha)$ for every $x \in X$ and $\alpha \in \Lambda$. Moreover, the sequence $\left(A_{[\alpha]_{n}}\right)_{n \geq 1}$ is decreasing, $\left\{\pi_{\mathcal{S}}(\alpha)\right\}=\bigcap_{n \geq 1} \overline{A_{[\alpha]_{n}}}$ and $\lim _{n \rightarrow \infty} \sup _{\alpha \in \Lambda_{n}} d\left(A_{\alpha}\right)=0$.

Proof The existence of the set $A(\mathcal{S})$ with the properties from the theorem was proved in [11]. As in the case of Theorem 2.4, the proof is almost as the proof of Theorem 4.1 from [16] with the exception of the fact that $\lim _{n \rightarrow \infty} \sup _{\alpha \in \Lambda_{n}} d\left(A_{\alpha}\right)=0$. As in the proof of Theorem 2.4, the sequence $\left(\sup _{\alpha \in \Lambda_{n}} d\left(A_{\alpha}\right)\right)_{n \geq 1}$ is decreasing; and therefore it is convergent to a number $l \geq 0$. We want to prove that $l=0$. Let us suppose that $l>0$. Since the metric space $(X, d)$ is uniform $\varepsilon^{\prime}$-chainable, there exists a natural number $N$ such that for every $x, y \in A$ there exists a chain $x_{0}=x, x_{1}, \ldots, x_{n}=y$ with the properties that $n+1 \leq N$ and $d\left(x_{i}, x_{i+1}\right)<\varepsilon^{\prime}$ for every $i \in\{0,1, \ldots, n-1\}$. To a set $B \subset A$ we associate the number

$$
\tilde{d}(B)=\sup _{x, y \in A} \inf \left\{\sum_{k=0}^{n-1} d\left(x_{k}, x_{k+1}\right): x_{0}=x, x_{1}, \ldots, x_{n}=y \text { is an } \varepsilon^{\prime} \text {-chain and } n+1 \leq N\right\} .
$$

It is clear that $\tilde{d}(A) \leq N \varepsilon^{\prime}, \tilde{d}(B) \geq d(B)$ and $\tilde{d}(B) \geq \tilde{d}\left(f_{i}(B)\right)$ for every set $B \subset A$ and $i \in I$. It follows that the sequence $\left(\sup _{\alpha \in \Lambda_{n}} \tilde{d}\left(A_{\alpha}\right)\right)_{n \geq 1}$ is decreasing and it is convergent to a number $\tilde{l} \geq l>0$. Let us note that $\frac{\tilde{l}}{N} \leq \frac{\tilde{d}(A)}{N} \leq \varepsilon^{\prime}$.

Since the family of functions $\left(f_{i}\right)_{i \in I}$ is $\varepsilon$-uniform Meir-Keeler for every $\varepsilon^{\prime \prime} \in(0, \varepsilon)$, there exist $\eta>0$ and $\lambda>0$ such that for every $i \in I$ and every $x, y \in X$ with the property that $d(x, y) \leq \varepsilon^{\prime \prime}+\eta$ we have $d\left(f_{i}(x), f_{i}(y)\right)<\varepsilon^{\prime \prime}-\lambda$. As a result, for every $\varepsilon^{\prime \prime} \in(0, \varepsilon)$, there exist $\delta>0$ and $\lambda^{\prime}>0$ such that $d\left(f_{i}(x), f_{i}(y)\right)<d(x, y)-\lambda^{\prime}$ for every $i \in I$ and every $x, y \in X$ with the property that $d(x, y) \in\left(\varepsilon^{\prime \prime}-\delta, \varepsilon^{\prime \prime}+\delta\right)$. To see this, we can take $\delta=\min (\lambda / 2, \eta)$ and $\lambda^{\prime}=\lambda / 2$. Indeed, if $d(x, y) \in\left(\varepsilon^{\prime \prime}-\delta, \varepsilon^{\prime \prime}+\delta\right)$, then $d(x, y) \leq \varepsilon^{\prime \prime}+\eta$ and therefore

$$
d\left(f_{i}(x), f_{i}(y)\right)<\varepsilon^{\prime \prime}-\lambda=\varepsilon^{\prime \prime}-\lambda / 2-\lambda / 2 \leq d(x, y)-\lambda / 2 .
$$

It follows that for every $\varepsilon_{1}, \varepsilon_{2} \in(0, \varepsilon)$ with $\varepsilon_{1}<\varepsilon_{2}$ there exists a number $\lambda>0$ such that $d\left(f_{i}(x), f_{i}(y)\right)<d(x, y)-\lambda$ for every $x, y \in X$ with the property that $d(x, y) \in\left[\varepsilon_{1}, \varepsilon_{2}\right]$ 
and every $i \in I$. In particular, there exists a number $\lambda>0$ such that $d\left(f_{i}(x), f_{i}(y)\right)<$ $d(x, y)-\lambda$ for every $x, y \in X$ with the property that $d(x, y) \in\left[\frac{\tilde{l}}{N+1}, \varepsilon^{\prime}\right]$ and every $i \in I$. Since $\lim _{n \rightarrow \infty} \sup _{\alpha \in \Lambda_{n}} \tilde{d}\left(A_{\alpha}\right)=\tilde{l}$, there exists $n_{0}$ such that for every $n \geq n_{0}$ we have $\sup _{\alpha \in \Lambda_{n}} \tilde{d}\left(A_{\alpha}\right)<\tilde{l}+\lambda / 2$.

Let us fix $\alpha \in \Lambda_{n_{0}}, i \in I$ and $x, y \in A_{i \alpha}$. Then there exist $\tilde{x}, \tilde{y} \in A$ such that $f_{i \alpha}(\tilde{x})=x$ and $f_{i \alpha}(\tilde{y})=y$. Since $f_{\alpha}(\tilde{x}), f_{\alpha}(\tilde{y}) \in A_{\alpha}$ and $\tilde{d}\left(A_{\alpha}\right)<\tilde{l}+\lambda / 2$, there exists an $\varepsilon^{\prime}$-chain in $X$, namely $x_{0}, x_{1}, \ldots, x_{n}$, such that $n+1 \leq N, f_{\alpha}(\tilde{x})=x_{0}, f_{\alpha}(\tilde{y})=x_{n}$ and $\sum_{k=0}^{n-1} d\left(x_{k}, x_{k+1}\right)<\tilde{l}+\lambda / 2$. We have two cases as follows:

(i) $\sum_{k=0}^{n-1} d\left(x_{k}, x_{k+1}\right) \leq \tilde{l}-\frac{\tilde{l}}{N+1}$;

(ii) $\sum_{k=0}^{n-1} d\left(x_{k}, x_{k+1}\right)>\tilde{l}-\frac{l}{N+1}$.

In the first case, we have

$$
\sum_{k=0}^{n-1} d\left(f_{i}\left(x_{k}\right), f_{i}\left(x_{k+1}\right)\right) \leq \sum_{k=0}^{n-1} d\left(x_{k}, x_{k+1}\right) \leq \tilde{l}-\frac{\tilde{l}}{N+1}
$$

In the second case, we note that there exists $k_{0} \in\{0,1, \ldots, n-1\}$ such that $d\left(x_{k_{0}}, x_{k_{0}+1}\right) \geq$ $\frac{1}{N}\left(\tilde{l}-\frac{\tilde{l}}{N+1}\right)=\frac{\tilde{l}}{N+1}$. Since $d\left(x_{k_{0}}, x_{k_{0}+1}\right) \in\left[\frac{\tilde{l}}{N+1}, \varepsilon^{\prime}\right]$, it follows that $d\left(f_{i}\left(x_{k_{0}}\right), f_{i}\left(x_{k_{0}+1}\right)\right)<d\left(x_{k_{0}}\right.$, $\left.x_{k_{0}+1}\right)-\lambda$ and

$$
\begin{aligned}
\sum_{k=0}^{n-1} d\left(f_{i}\left(x_{k}\right), f_{i}\left(x_{k+1}\right)\right) & =d\left(f_{i}\left(x_{k_{0}}\right), f_{i}\left(x_{k_{0}+1}\right)\right)+\sum_{k=0 ; k \neq k_{0}}^{n-1} d\left(f_{i}\left(x_{k}\right), f_{i}\left(x_{k+1}\right)\right) \\
& \leq d\left(x_{k_{0}}, x_{k_{0}+1}\right)-\lambda+\sum_{k=0 ; k \neq k_{0}}^{n-1} d\left(x_{k}, x_{k+1}\right) \\
& =-\lambda+\sum_{k=0}^{n-1} d\left(x_{k}, x_{k+1}\right) \leq-\lambda+\tilde{l}+\lambda / 2=\tilde{l}-\lambda / 2 .
\end{aligned}
$$

Therefore $\tilde{d}\left(A_{i \alpha}\right) \leq \tilde{l}-\max \left(\lambda / 2, \frac{\tilde{l}}{N+1}\right)$ for every $\alpha \in \Lambda_{n_{0}}, i \in I$, which leads to the following contradiction:

$$
\tilde{l} \leq \sup _{\alpha \in \Lambda_{n_{0}+1}} \tilde{d}\left(A_{\alpha}\right) \leq \tilde{l}-\max \left(\lambda / 2, \frac{\tilde{l}}{N+1}\right) .
$$

This ends the proof in the first case. The second case can be reduced to the first one in a similar manner as we have done in the proof of Theorem 3.2.

The main result of this section is contained in the following.

Theorem 4.11 Let $\mathcal{S}=\left(f_{i}\right)_{i \in I}$ be an IIFS on the complete metric space $(X, d)$ and $\varepsilon>\varepsilon^{\prime}>0$. Then there exists a unique fixed point $\pi_{0}$ of $H_{\mathcal{S}}$ and $d_{\infty}\left(H_{\mathcal{S}}^{[n]}(f), \pi_{0}\right) \rightarrow 0$ for every $f \in$ $\mathrm{CB}(\Lambda, X)$ provided that one of the following two conditions is fulfilled:

(i) the family of functions $\left(f_{i}\right)_{i \in I}$ is uniform $\varepsilon$-Meir-Keeler and $X$ is uniform $\varepsilon^{\prime}$-chainable;

(ii) $(X, d)$ is a compact $\varepsilon^{\prime}$-chainable metric space, $I$ is a finite set and all the functions $f_{i}$ are $\varepsilon$-contractive.

Moreover, $\pi_{0} \circ \tau_{i}=f_{i} \circ \pi_{0}$ for every $i \in I$; and consequently $\pi_{0}=\pi$. 
Proof We start with the first case (the family of functions $\left(f_{i}\right)_{i \in I}$ is uniform $\varepsilon$-Meir-Keeler and $X$ is uniform $\varepsilon^{\prime}$-chainable). Let us consider $\varepsilon^{\prime \prime} \in(0, \varepsilon)$. Then there exist $\eta>0$ and $\lambda>0$ such that for every $x, y \in X$ with the property that $d(x, y)<\varepsilon+\eta$ we have $d\left(f_{i}(x), f_{i}(y)\right)<\varepsilon-\lambda$. If $f, g \in \mathrm{CB}(\Lambda, X)$ have the property that $d_{\infty}(f, g)<\varepsilon+\eta$, then with the same argument as in Proposition 3.1 point (iv) one can prove that $d_{\infty}\left(H_{\mathcal{S}}(f), H_{\mathcal{S}}(g)\right)<\varepsilon-\lambda / 2$. This implies that $H_{\mathcal{S}}$ is $\varepsilon$-Meir-Keeler. $\left(\mathrm{CB}(\Lambda, X), d_{\infty}\right)$ is a complete metric space since $(X, d)$ is so and it is $\varepsilon^{\prime}$-chainable from Proposition 4.7. Therefore we can apply Theorem 4.9 to obtain the desired result.

From the fact that $H_{\mathcal{S}}\left(\pi_{0}\right)=\pi_{0}$ we infer that $\pi_{0} \circ \tau_{i}(\alpha)=\pi_{0}(i \alpha)=H_{\mathcal{S}} \circ \pi_{0}(i \alpha)=f_{i}\left(\pi_{0}(\alpha)\right)$ for every $\alpha \in \Lambda$ and $i \in I$, i.e., $\pi_{0} \circ \tau_{i}=f_{i} \circ \pi_{0}$ for every $i \in I$.

For $\alpha, \beta \in \Lambda$, we have $[\alpha]_{n} \beta \rightarrow \alpha$ in $\left(\Lambda, d_{\Lambda}\right)$; and consequently $\pi_{0}\left([\alpha]_{n} \beta\right) \rightarrow \pi_{0}(\alpha)$. But $\pi_{0}\left([\alpha]_{n} \beta\right)=\pi_{0} \circ \tau_{[\alpha]_{n}}(\beta)=f_{[\alpha]_{n}} \circ \pi_{0}(\beta)$, and from Theorem 4.10 we infer that $f_{[\alpha]_{n}} \circ \pi_{0}(\beta) \rightarrow$ $\pi_{\mathcal{S}}(\alpha)$. Therefore $\pi_{0}=\pi_{\mathcal{S}}$.

In the second case, namely $(X, d)$ is a compact $\varepsilon^{\prime}$-chainable metric space, $I$ is a finite set and all the functions $f_{i}$ are $\varepsilon$-contractive, we consider the function $\phi:[0, \varepsilon) \rightarrow \mathbb{R}$ defined by $\phi(r)=\sup \left\{d\left(f_{i}(x), f_{i}(y)\right): i \in I\right.$ and $\left.d(x, y) \leq r\right\} . \phi$ is increasing, right continuous and $\phi(r)<r$ for every $r \in(0, \varepsilon)$. If $\varepsilon^{\prime \prime} \in\left(0, \varepsilon^{\prime}\right)$, there exists $\eta>0$ such that $\varepsilon^{\prime \prime}+\eta<\varepsilon$ and $\phi(r)<\frac{\phi\left(\varepsilon^{\prime \prime}\right)+\varepsilon^{\prime \prime}}{2}$ for every $r \in\left[\varepsilon^{\prime \prime}, \varepsilon^{\prime \prime}+\eta\right)$. It follows that $\phi\left(d\left(f_{i}(x), f_{i}(y)\right)\right) \leq \varepsilon^{\prime \prime}-\frac{\varepsilon^{\prime \prime}-\phi\left(\varepsilon^{\prime \prime}\right)}{2}<\varepsilon^{\prime \prime}-\frac{\varepsilon^{\prime \prime}-\phi\left(\varepsilon^{\prime \prime}\right)}{4}$ for every $r \in\left[0, \varepsilon^{\prime \prime}+\eta\right)$. In this way the second case can be reduced to the first one.

\section{Remarks and examples}

It is known that every bounded set of a metric space is the attractor of an IIFS containing constant functions. We will compute the function $H_{S}$ for such an IIFS.

Example 5.1 Let $(X, d)$ be a complete metric space and $A \in B(X)$. For $a \in X$, $f_{a}$ will denote the constant function with value $a$, that is, $f_{a}: X \rightarrow X$ and $f_{a}(x)=a$ for every $x \in X$. Then $A$ is the attractor of the IIFS $\mathcal{S}=\left(X,\left(f_{a}\right)_{a \in A}\right)$. Also $A$ is the attractor of the IIFS $\mathcal{S}_{B}=\left(X,\left(f_{a}\right)_{a \in B}\right)$ for any dense set $B$ in $A$. If $A$ is separable and $B$ is a countable dense set in $A$, then $A$ is the attractor of the CIFS (countable IFS) $\mathcal{S}_{B}=\left(X,\left(f_{a}\right)_{a \in B}\right)$. This happens, in particular, for any compact set $A$. We have

$$
H_{S}(f)(\alpha)=H_{\mathcal{S}}(f)(\alpha)=f_{[\alpha]_{1}} \circ f \circ R(\alpha)=[\alpha]_{1} .
$$

We try now to compute the iterations of $H_{\mathcal{S}}$ in a general case.

Remark 5.2 Let $\mathcal{S}=\left(f_{i}\right)_{i \in I}$ be an IIFS on the complete metric space $(X, d)$ and $\alpha=$ $\alpha_{1} \alpha_{2} \cdots \in \Lambda$. Then

$$
\begin{aligned}
H_{\mathcal{S}}^{[2]}(f)(\alpha) & =H_{\mathcal{S}}\left(H_{\mathcal{S}}(f)\right)(\alpha)=f_{[\alpha]_{1}} \circ H_{\mathcal{S}}(f) \circ R(\alpha) \\
& =f_{[\alpha]_{1}} \circ H_{\mathcal{S}}(f)(R(\alpha))=f_{[\alpha]_{1}} \circ f_{[R(\alpha)]_{1}} \circ f \circ R^{[2]}(\alpha) \\
& =f_{\alpha_{1}} \circ f_{\alpha_{2}} \circ f \circ R^{[2]}(\alpha)=f_{\alpha_{1} \alpha_{2}} \circ f \circ R^{[2]}(\alpha) .
\end{aligned}
$$

By induction it can be proved that $H_{\mathcal{S}}^{[n]}(f)(\alpha)=f_{[\alpha]_{n}} \circ f \circ R^{[n]}(\alpha)$.

In particular if the function $f$ is constant, namely $f(\alpha)=b$ for $b \in X, H_{\mathcal{S}}^{[n]}(f)(\alpha)=f_{[\alpha]_{n}}(b)$.

We consider now the case when $(X,\|\cdot\|)$ is a Banach space and $\mathcal{S}=\left(X,\left(f_{i}\right)_{i \in I}\right)$ is an IIFS formed by affine transformations. That is, $f_{i}=A_{i}+b_{i}$, where $A_{i}$ are linear and continuous 
operators $A_{i}: X \rightarrow X$ with $\left\|A_{i}\right\|<c<1$ and $b_{i} \in X$ for every $i \in I$ are such that the set $\left\{b_{i}: i \in I\right\}$ is bounded. By induction one can see that

$$
f_{i_{1} i_{2} \cdots i_{n}}(x)=f_{i_{1}} \circ f_{i_{2}} \circ \cdots \circ f_{i_{n}}(x)=A_{i_{1}} A_{i_{2}} \cdots A_{i_{n}} x+\sum_{i=1}^{n} A_{i_{1}} A_{i_{2}} \cdots A_{i_{i-1}} b_{i}
$$

Therefore

$$
H_{\mathcal{S}}^{[n]}(f)(\alpha)=f_{[\alpha]_{n}} \circ f \circ R^{[n]}(\alpha)=A_{i_{1}} A_{i_{2}} \cdots A_{i_{n}} f \circ R^{[n]}(\alpha)+\sum_{i=1}^{n} A_{i_{1}} A_{i_{2}} \cdots A_{i_{i-1}} b_{i} .
$$

In particular, if the function $f$ is constant, namely $f(\alpha)=b$ for $b \in X$,

$$
H_{\mathcal{S}}^{[n]}(f)(\alpha)=A_{i_{1}} A_{i_{2}} \cdots A_{i_{n}} b+\sum_{i=1}^{n} A_{i_{1}} A_{i_{2}} \cdots A_{i_{i-1}} b_{i}
$$

From Theorem 2.4 it follows that $\pi_{\mathcal{S}}(\alpha)=\sum_{i \geq 1} A_{i_{1}} A_{i_{2}} \cdots A_{i_{i-1}} b_{i}$, where $\pi_{\mathcal{S}}$ is the canonical projection. Then

$$
\begin{aligned}
d_{\infty}\left(H_{\mathcal{S}}^{[n]}(f), \pi_{\mathcal{S}}\right) & =\sup _{\alpha \in \Lambda}\left\|H_{\mathcal{S}}^{[n]}(f)(\alpha)-\pi_{\mathcal{S}}(\alpha)\right\| \\
& =\sup _{\alpha \in \Lambda}\left\|A_{i_{1}} A_{i_{2}} \cdots A_{i_{n}} f \circ R^{[n]}(\alpha)-\sum_{i \geq n+1} A_{i_{1}} A_{i_{2}} \cdots A_{i_{i-1}} b_{i}\right\| \\
& \leq \sup _{\alpha \in \Lambda}\left(c^{n}\|f(\alpha)\|+\frac{c^{n}}{1-c} \sup _{i \in I}\left\|b_{i}\right\|\right) \\
& =c^{n}\left(\sup _{\alpha \in \Lambda}\|f(\alpha)\|+\frac{1}{1-c} \sup _{i \in I}\left\|b_{i}\right\|\right) .
\end{aligned}
$$

Example 5.3 Let $I$ be a nonvoid set, $j \notin I,(X,\|\cdot\|)$ be a Banach space and $\mathcal{S}=\left(X,\left(f_{i}\right)_{i \in I \cup\{j\}}\right)$ be an IIFS formed by affine transformations such that $f_{i}$ are constant functions for $i \in I$ $\left(f_{i}(x)=b_{i}\right)$ and $f_{j}$ is affine functions of the form $f_{j}(x)=a x+b$, where $a \in(-1,1)$ and $b \in X$. Then for an infinite word $\alpha=i_{1} i_{2} \cdots i_{m} \cdots, H_{\mathcal{S}}^{[n]}(f)(\alpha)$ is $b_{1}$ if $i_{1} \neq j, a^{k} b_{i_{k+1}}+\sum_{i=1}^{k} a^{i-1} b$ if $i_{1}=i_{2}=\cdots=i_{k}=j, k \leq n-1$ and $i_{k+1} \neq j$ and $a^{n} f \circ R^{[n]}(\alpha)+\sum_{i=1}^{n} a^{i-1} b$ if $i_{1}=i_{2}=\cdots=i_{n}=j$. Also $\pi_{\mathcal{S}}(\alpha)$ is $b_{1}$ if $i_{1} \neq j, a^{k} b_{i_{k+1}}+\sum_{i=1}^{k+1} a^{i-1} b$ if $i_{1}=i_{2}=\cdots=i_{k}=j$ and $i_{k+1} \neq j$ and $\frac{b}{1-a}$ if $i_{1}=i_{2}=\cdots=i_{m}=\cdots=j$. Then

$$
\begin{aligned}
& \left\|H_{\mathcal{S}}^{[n]}(f)(\alpha)-\pi_{\mathcal{S}}(\alpha)\right\| \\
& \quad= \begin{cases}0 & \text { if there exists } k<n \text { such that } i_{k} \neq j, \\
a^{n} f \circ R^{[n]}(\alpha)-a^{k} b_{i_{k+1}}-\sum_{i=n+1}^{k} a^{i-1} b & \text { if } i_{1}=\cdots=i_{k}=j, k \geq n \text { and } i_{k+1} \neq j, \\
a^{n} f \circ R^{[n]}(\alpha)-\sum_{i \geq n+1} a^{i-1} b & \text { if } i_{1}=i_{2}=\cdots=i_{m}=\cdots=j .\end{cases}
\end{aligned}
$$

If $f$ is a constant function with value $d$, then

$$
\left\|H_{\mathcal{S}}^{[n]}(f)-\pi_{\mathcal{S}}\right\|=\sup _{k \geq n}|a|^{n}\left\|d-b \sum_{i=n+1}^{k} a^{i-n-1}-a^{k-n} b_{i_{k+1}}\right\| .
$$


Example 5.4 (The Cantor set) Let $(X,\|\cdot\|)$ be a Banach space, $b \in X \backslash\left\{0_{X}\right\}$ and an IFS $\mathcal{S}=\left(X,\left(f_{i}\right)_{i \in\{-1,1\}}\right)$, where $f_{-1}(x)=\frac{x-2 b}{3}$ and $f_{1}(x)=\frac{x+2 b}{3}$. The attractor of $\mathcal{S}$ is homeomorphic with the Cantor set. Then, for an infinite word $\alpha=i_{1} i_{2} \cdots i_{m} \cdots, H_{\mathcal{S}}^{[n]}(f)(\alpha)=f_{[\alpha]_{n}} \circ f \circ$ $R^{[n]}(\alpha)=\frac{1}{3^{n}} f \circ R^{[n]}(\alpha)+\sum_{i=1}^{n} \frac{(-1)^{i} i_{i}{ }^{i}}{3^{i}}$. If $f$ is a constant function with value $c$, then $H_{\mathcal{S}}^{[n]}(f)(\alpha)=$ $\frac{1}{3^{n}} c+\sum_{i=1}^{n} \frac{(-1)^{i} 2^{i}}{3^{i}} b$.

It is an open problem to find contractive conditions which are inherited by some of the operators $F_{\mathcal{S}}, H_{\mathcal{S}}$ and $M_{\mathcal{S}}$ from the constitutive functions of the IIFS $\mathcal{S}$ or contractive conditions which assure us that some of the operators $F_{\mathcal{S}}, H_{\mathcal{S}}$ and $M_{\mathcal{S}}$ have a unique fixed point. It is also an open problem to extend the above results to different generalizations of IFSs (such as GIFSs).

Competing interests

The author declares that he has no competing interests.

\section{Author's contributions}

The author completed the paper himself. The author read and approved the final manuscript.

\section{Acknowledgements}

We want to thank the referees whose generous and valuable remarks and comments brought improvements to the paper and enhanced clarity.

Received: 22 October 2014 Accepted: 8 May 2015 Published online: 27 May 2015

\section{References}

1. Miculescu, R, Mihail, A: Lipscomb's space $\omega^{A}$ is the attractor of an infinite IFS containing affine transformations of R(A).Proc Am Math Soc 136, 587-592 (2008)

2. Miculescu, R, Mihail, A: Lipscomb's $L(A)$ space fractalized in $\mathbb{P}(A)$. Mediterr. J. Math. 9, 515-524 (2012)

3. Perry, JC: Lipscomb's universal space is the attractor of an infinite iterated function system. Proc. Am. Math. Soc. 124 2479-2489 (1996)

4. Moran, P: Additive functions on intervals and Hausdorff measure. Proc. Camb. Philos. Soc. 42, 15-23 (1946)

5. Hutchinson, JE: Fractals and self similarity. Indiana Univ. Math. J. 30, 713-747 (1981)

6. Barnsley, MF: Fractals Everywhere. Academic Press, Boston (1993)

7. Andres, J, Fišer, J, Gabor, G, Leśniak, K: Multivalued fractals. Chaos Solitons Fractals 24, 665-700 (2005)

8. Mihail, A, Miculescu, R: Applications of fixed point theorems in the theory of generalized IFS. Fixed Point Theory Appl. 2008, Article ID 312876 (2008)

9. Mihail, A, Miculescu, R: Generalized IFSs on noncompact spaces. Fixed Point Theory Appl. 2010, Article ID 584215 (2010)

10. Secelean, NA: Generalized iterated function systems on the space ${ }^{\infty}$ (X). J. Math. Anal. Appl. 410, $847-858$ (2014)

11. Dumitru, D: Attractors of infinite iterated function systems containing contraction type functions. An. Ştiinţ. Univ. 'Al.I. Cuza' laşi, Mat. 59, 281-298 (2013)

12. Secelean, NA: Iterated function systems consisting of F-contractions. Fixed Point Theory Appl. 2013, 277 (2013)

13. Dumitru, D: Arcwise connected attractors of infinite iterated function systems. An. Ştiinţ. Univ. 'Ovidius' Constanţa 22 91-98 (2014)

14. Kigami, J: Analysis on Fractals. Cambridge University Press, Cambridge (2001)

15. Barnsley, MF: Transformation between attractors of hyperbolic iterated function systems (2007). arXiv:math/0703398v1 [math.DS]

16. Mihail, A, Miculescu, R: The shift space for an infinite iterated function system. Math. Rep. 61, 21-32 (2009)

17. Meir, A, Keeler, E: A theorem on contraction mappings. J. Math. Anal. Appl. 28, 326-329 (1969) 